\title{
7 TIC's para jóvenes indígenas: una experiencia mapuche en la creación colectiva de contenidos culturales digitales
}

Leonor Slavsky ${ }^{1}$

os años que siguieron al quinto centenario de la llegada de los Lespañoles al continente americano modificaron profundamente el panorama indígena en nuestro país y en América, adquiriendo una visibilidad que en la Argentina hasta entonces no tenía. La modificación del artículo constitucional (Art. 75 inc. 17) y la activa participación de los pueblos indígenas en este logro, produjeron una revitalización que se traduce en movimientos de retradicionalización (la búsqueda conciente y sistemática en el pasado de elementos identificatorios, incluyendo la lengua) y de reetnización (el reagrupamiento de individuos que reivindican su ancestría indígena), produciendo en el escenario público la reaparición de grupos étnicos que la etnografía clásica daba por desaparecidos, tales como los huarpes, onas, charrúas y tonocotés.

También ha cambiado el eje del debate y los reclamos hacia el gobierno nacional, los académicos y la sociedad en general. Ya no se trata de crear legislación ${ }^{2}$, sino de avanzar hacia su aplicación efectiva y garantizar la participación indígena en los temas que los involucran. Aparecen temas nuevos, como el reconocimiento de los derechos de propiedad intelectual, la biodiversidad, la comunicación y los derechos consuetudinarios, pero el "núcleo duro" de los derechos indígenas (respeto a la identidad étnica y cultural, tierra, educación, personería jurídica de las comunidades) ya está sancionado, si bien su aplicación en la práctica todavía es muy precaria. Estos derechos

1 Investigadora del Instituto Nacional de Antropología y Pensamiento Latinoamericano, Secretaría de Cultura de la Nación. Lic. en Ciencias Antropológicas, Mag. en Metodología de la Investigación. Coordinadora del proyecto Tecnologías de la Información y la Comunicación para Jóvenes Mapuche.

2 Art. 75 inc. 17 de la Constitución Nacional, Convenio 169 de la Organización Internacional del Trabajo, ley 23.302 (a pesar de sus falencias), Constituciones y leyes provinciales. 
se encuentran íntimamente relacionados, se implican los unos a los otros, e involucran el derecho a la participación y a la autodefinición.

El derecho a la participación es claramente un reclamo político vinculado con el ejercicio del poder. Bonfil Batalla, en su teoría del control cultural, ponía este concepto en el centro de su definición de grupo étnico:

"Conjunto relativamente estable de individuos que mantiene una continuidad histórica porque se reproduce biológicamente y porque sus miembros establecen entre sí vínculos de identidad social distintiva a partir de que se asumen como una unidad política (real o virtual, presente o pasada) que tiene derecho exclusivo al control de un universo de elementos culturales que consideran propio" (Bonfil Batalla, 1989).

Estos elementos culturales, lejos de estar inmóviles en el tiempo tienen una dinámica de cambio que incluye la apropiación de elementos culturales ajenos al grupo, la creación de nuevos y la resignificación de contenidos del pasado. El punto clave está en la capacidad de decisión del conjunto social sobre cuáles son los contenidos culturales significativos en el momento presente y la capacidad propia de producirlos y reproducirlos.

Los reclamos por la autodefinición (también de carácter político), confrontan no sólo con el Estado sino también con la autoridad académica, en una puja por la atribución de "autenticidad" de las culturas indígenas. Citando a Robert Paine:

"Por largo tiempo, el 'ser' aborigen ha sido una construcción subalterna atribuida por el mundo colonial a los indígenas de acuerdo a su propio entendimiento -una comprensión del sí mismo- a través de un retrato contrastivo con el mundo aborigen. Sin embargo hoy, las sociedades aborígenes están comprometidas en una autodefinición cara a cara con el mundo colonial, y la autenticidad se ha transformado entre ellos en un tema bajo intenso escrutinio".

Esto significa que la vieja dicotomía "colonial" que clasificaba a la gente como civilizada o incivilizada como única opción, está desapareciendo con la autoatribución de autenticidad. Los pueblos están reclamando su aboriginalidad, tal como ellos la entienden. En síntesis, están reclamando (y reformulando en el proceso) su propio 
yo. La actual politización de los temas sobre la identidad aborigen y sus derechos es fundamentalmente una cuestión de redefinición y redistribución. Los esfuerzos de autoatribución entre las poblaciones aborígenes, los conduce a interrogarse: “¿Quiénes somos? ¿Cuál es nuestra tradición? ¿Cuáles de nuestras tradiciones deberían restituirse o modificarse? y no simplemente a proclamar su emancipación" (Paine 2000: 77-78).

Este planteo no se limita al campo de las políticas públicas, también involucra el terreno de la investigación, cuestionando la autoridad antropológica. Las posturas extremas se presentan en los Estados U nidos, dentro de posiciones multiculturalistas que plantean la incapacidad del investigador ajeno a una cultura, para llevar a cabo su trabajo, tanto en el campo de la antropología social como de la arqueología:

"las tribus indígenas demandan en forma creciente que el experto sea el propio indígena, y sostienen que en muchas circunstancias el no indígena es irrelevante, no deseado y a quien se le debe negar el acceso a la información" (Klesert y Benallie 1994).

Esta situación implica un replanteo en varios sentidos. ¿Se investiga sobre los indígenas, con los indígenas o para los indígenas? ¿Se gestiona con o para ellos? El "sobre" define la actividad académica clásica para la cual los indígenas son un objeto de estudio, el "con" las posturas del indigenismo participativo, el "para" puede implicar o bien una posición de defensa de derechos participativa o no (el mundo anglosajón lo denomina advocacy), o bien la realización de trabajos a solicitud de las comunidades u organizaciones, poniendo al servicio de los mismos conocimientos y metodologías propios del campo socio-antropológico, pero respetando sus objetivos y límites. ¿Se puede trabajar sobre, con y para simultáneamente? (Slavsky 2004).

El correo electrónico y la Internet no jugaron un papel menor en estos procesos. Agilizaron la fluidez en los contactos y estimularon la proyección de líderes locales a la arena nacional e internacional, instalaron movimientos políticos (como en el caso del Ejército Zapatista de Liberación), al mismo tiempo que presentan a través de distintas páginas en la W eb interesantes paisajes culturales que cabal gan entre la tradición y la modernidad. 


\section{La Sociedad de la Información y los pueblos indígenas}

La concepción actual de la Sociedad de la Información es influjo de la obra de varios autores, entre ellos Daniel Bell (1977), quien estimaba que en razón del progreso técnico, las actividades de tratamiento de la información son inducidas a reemplazar las actividades industriales de manipulación de la materia que el siglo pasado había tomado el lugar de las actividades agrícolas. El avance de la industria se haría cuando la información sustituyera a la producción pesada. Las consecuencias generarían que el valor-saber sustituyera el valortrabajo, y que ocurrieran profundos cambios en la composición de las clases sociales, con el incremento de una clase media asalariada y provista con un alto nivel de formación.

También el sociólogo Yoneji M asuda (1984), analizó los cambios en la sociedad industrial, básicamente cómo los medios de generación de riqueza se trasladan de los sectores industriales a los sectores de servicios, y supone que en las sociedades modernas, la mayor parte de los empleos ya no estarán asociados a las fábricas de productos tangibles, sino a la generación, almacenamiento y procesamiento de todo tipo de información. Los sectores relacionados con las tecnologías de la información y la comunicación (TIC), desempeñan un papel particularmente importante dentro de este esquema.

De acuerdo con Claudia Benassini Félix (2003), el concepto "Sociedad de la Información", ha atravesado por lo menos tres etapas reflexivas:

En el primero, surgen intentos por caracterizar este nuevo panorama partiendo de nociones previas procedentes de diversas disciplinas, donde la sociología y la economía tuvieron un lugar protagónico. Prevalecen, sin embargo, dos posiciones antagónicas entre las que media un continuum de alternativas producto de las diferencias generadas por la propia Sociedad de la Información, tanto entre contextos socioculturales como entre las experiencias individuales y grupales. La primera $^{3}$ reflexiona desde diversas disciplinas sobre las implicaciones del nuevo panorama, particularmente de las nuevas maneras de relación con los otros -en tanto alteridades- así como en la inevitable brecha

3 De acuerdo con Armand Mattelart, "la cultura global" corresponde al sistema de dominación que con brutal violencia ha impuesto Estados Unidos en el mundo, y las avanzadas tecnologías de la información y las comunicaciones están subordinadas a los propósitos de control y expansión de la hegemonía que ejercen las clases dominantes. 
digital. La segunda, optimista, se centra en las bondades de las nuevas posibilidades comunicativas y en las que están por venir, aunque identificando parte de los obstáculos.

En un segundo momento -que necesariamente se traslapa con el primero, en tanto construcción de la noción-, la evolución de las comunicaciones se caracteriza, en un extremo, por un optimismo casi desbordante. Las comunicaciones mediadas por la computadora han abierto infinidad de perspectivas a los usuarios. El correo electrónico y la Internet se han popularizado, lo mismo que la disponibilidad de un equipo de computación, tanto personal como cibercafés. Pero en el otro extremo, subsisten no sólo las diferencias marcadas tanto por la brecha digital ${ }^{4}$ como por otros fenómenos, en cuyo origen no sólo está la lucha por el poder, sino contradicciones axiológicas que confunden las perspectivas.

El tercer momento, que también se traslapa con el primero y el segundo, supone abandonar la percepción homogénea y abstracta de la Sociedad de la Información, para volver la mirada hacia sus habitantes, en tanto público de un nuevo medio de comunicación. Implica la toma de conciencia de que la Sociedad de la Información está conformada por comunidades de sentido, justamente producto de las comunicaciones mediadas por computadora. Comunidades de sentido que generan nexos de pertenencia y cierto tipo de rituales de acercamiento al otro, más allá del puro encuentro virtual y en torno a los intereses comunes tan diversos como su constitución (Benassini 2003).

Tampoco ha estado ausente el análisis sobre los pueblos indígenas en la sociedad de la información. Particularmente interesantes son los producidos por del Alamo (2003) y Hernández y Calcagno (2003). En ambos trabajos se analizan los desafíos, las oportunidades y las propuestas de las TIC. Entre las primeras, se señalan altos niveles de prejuicio en las oficinas estatales, ONG's y amplios sectores de las sociedades nacionales; bajos niveles de alfabetización y de alfabetismo informático; procesos intracomunitarios que dificultan el desarrollo de estas tecnologías; elevados costos de la infraestructura tecnológica, asociados al aislamiento geográfico y la carencia de servicios de infraestructura básica; expansión de las Tic's orientada según la lógica del mercado; predominio del inglés en el campo tecnológico. Las oportunidades se centran en la experiencia histórica de los pueblos indígenas

4 Este fenómeno se refiere a todos aquellos sectores que permanecen por diversas razones, al margen de los beneficios y ventajas asociados a las TIC. 
en la apropiación exitosa de bienes culturales de la sociedad gl obal; en el ascenso del movimiento étnico a nivel continental; en la presencia de liderazgo indígena de un nuevo tipo; un mayor reconocimiento de la sociedad global del papel de los pueblos indígenas en la sustentabilidad del desarrollo; experiencias de autogestión exitosas; creciente aumento del nivel educativo de la población indígena (entre los que se cuentan no pocos profesionales); aumento del capital social de las comunidades. Proponen la implementación de programas específicos de acceso, diseñados a partir de diagnósticos integrales realizados con la participación de las comunidades indígenas y respetuosas de sus estructuras sociales, culturales y económicas; articulación con programas integrales de largo plazo que contemplen inversión estratégica, para proveer de servicios básicos y promuevan la gestión autónoma de las comunidades; regulación jurídica de la participación del sector privado y apoyo del sector público, cuando el mercado y su dinámica no pueda aportar soluciones efectivas para combatir la desigualdad social; implementación de programas para explorar de manera intracomunitaria la forma en que las Tic's serán incorporadas, partiendo de la premisa que pueden optar por no utilizarlas.

Los propios pueblos indígenas y sus organizaciones forman parte del debate. En distintos foros internacionales discutieron su incorporación a la sociedad de la información, estableciendo las pautas de la misma. En el Foro Mundial de los Pueblos Indígenas y la Sociedad de Información, en diciembre del 2003, los representantes de las comunidades indígenas realizaron una declaración basada en los principios de igualdad en la diversidad y pleno respeto a los derechos humanos:

"La comunicación y la información son una práctica milenaria y cotidiana de los pueblos indígenas y debe ser analizada desde la perspectiva de la diversidad cultural, para evitar enfoques unilaterales que privilegian aspectos puramente tecnológicos e instrumentales, mientras se soslayan los contenidos, los sentimientos y los valores que son esenciales para la comunicación humana. Alertamos que la visión de una Sociedad del futuro cuya lógica de construcción esté basada en una competencia de mercado por el uso y acceso a nuevas tecnologías de la Información, conlleva el grave riesgo de desvirtuar el sentido de la comunicación humana. El paradigma de una Sociedad de la Información basada principalmente en el acceso universal a las TIC, significa ignorar las desigualdades estructurales cuyas causas no son de orden tec- 
nológico o de infraestructura, sino que responden a modelos de dominación excluyentes basados en el interés privado de corporaciones transnacionales, que concentran un gran poder tecnológico y que intentan generalizarlos por medio del mercado como única vía para el desarrollo humano"s.

En el II Encuentro sobre Conectividad y Poblaciones Indígenas, realizado en Ottawa en marzo de 2005, se decidió establecer una red de comunicación norte-sur para compartir experiencias; especificar estrategias para superar el desafío de la conectividad; utilizar las propias tecnologías para vencer los inconvenientes y crear contenidos culturales propios sin interferencias ni distorsiones ideológico-culturales ${ }^{6}$.

Con estas premisas asistieron a la II Fase de la Cumbre Mundial sobre la Sociedad de la Información (noviembre 2005) que incluyó en su carta de Compromiso:

"22. En la evolución de la Sociedad de la Información, se debe prestar una atención especial a la situación particular de los pueblos indígenas, así como a la preservación de su patrimonio y de su legado cultural".

Y en la Agenda de Túnez para la Sociedad de la Información, entre las líneas de acción:

"C8. Diversidad e identidad culturales, diversidad lingüística y contenido local"

\section{Proyecto Tecnologías de la Información y la Comunicación para Jóvenes Mapuche}

En el contexto descrito se inscribe el Proyecto "Tecnologías de la Información y la Comunicación para Jóvenes Mapuche", concebido como un proyecto de gestión participativa público-privado, con la participación del Instituto Nacional de Antropología y Pensamiento Latinoamericano de la Secretaría de Cultura de la Nación, el Programa

5 http://weblog.educ.ar/infosoc/archives/000559.php http://www.rebelion.org/medios/031210dec.htm www.aboriginalcanada.gc.ca/international.html

6 www.un.org/esa/socdev/unpfii/news/WSIS/newsottawaeventsp.htm

7 www.itu.int/wsis/index-es.html 
de Educación Intercultural Bilingüe del Ministerio de Educación, Ciencia y Tecnología de la Nación y el Servicio U niversitario Mundial, con el financiamiento del Instituto de Conectividad de las Américas del gobierno de Canadá.

Sus objetivos apuntan a lograr la apropiación de las tecnologías de la información y la comunicación por parte de las comunidades del pueblo mapuche, con el fin de fortalecer, resignificar y transmitir su conocimiento tradicional y promover a la gestión de su propio desarrollo; establecer conexión entre comunidades localizadas en zonas urbanas, semi urbanas y rurales; incentivar a los jóvenes a permanecer o regresar a sus comunidades de origen, promoviendo iniciativas educativas, culturales y económicas de interés comunitario; afianzar la presencia y visibilidad de las comunidades del Pueblo Mapuche, en diversos espacios sociales, foros de debate e intercambio de información y garantizar la comunicación y el intercambio de información entre distintos pueblos indígenas del país y del continente.

Los principios básicos para la acción son:

Consenso y participación: Supusimos un amplio acuerdo de los actores involucrados para impulsarlo, ejecutarlo y asegurar la participación de las comunidades en los ámbitos de toma de decisiones sobre el proyecto.

G radualidad: La tarea requeriría un proceso de evaluación y ajuste continuo de las acciones; en la medida en que los actores locales ganasen capacidades para asumir plenas responsabilidades, se iría ampliando el campo de actividades posibles.

Interculturalidad: Partimos del reconocimiento de la interacción entre sociedades diferentes que dialogan en mutuo respeto para el logro de los objetivos propuestos.

Las tareas a realizar fueron: proveer de acceso e infraestructura, promover el desarrollo de contenido local para una página de Internet y publicación de contenidos, estimular la búsqueda de oportunidades en la Red para el desarrollo con identidad, y capacitar a jóvenes de los nodos en alfabetización digital y uso inteligente de la Web.

Para lograr estos objetivos optamos por una metodología participativa y descentralizada, creando nodos en el interior y en Buenos Aires que enlazaran ciudades de escala pequeña, mediana y grande. En una primera instancia se eligió Bariloche (Río Negro) y El Maitén (Chubut) con sus comunidades aledañas y población urbana. Luego 
se instalaron otros nodos en Esquel, Viedma (comunidad M ongell Mamuel) y Bahía Blanca (Ruka Kimún) ${ }^{8}$.

Se realizaron reuniones comunitarias en cada uno de los nodos para explicar el proyecto, poner a los mayores en contacto con Internet y lograr consenso. Partimos del supuesto que los jóvenes tendrían una alfabetización digital básica, producto de la capacitación escolar y los cibercafés. Esta presunción resultó errada en la mayoría de los casos, pues si bien la mayoría de las escuelas poseen computadoras provistas por el Ministerio de Educación, casi todas se encuentran desaprovechadas por falta de actualización en los equipos y por dificultades para la conexión a la red (en algunos casos por imposibilidad de acceso, en otras por falta de pago por parte de las autoridades locales).

A pesar de una resistencia inicial por parte de los mayores, cuando pudieron acceder a la navegación de otros sitios indígenas en general y mapuches en particular, visualizaron las potencialidades de la W eb para el fortalecimiento de la cultura propia y la transmisión de conocimientos a la generación más joven. Estas mismas reuniones fueron aprovechadas para seleccionar los equipos de trabajo, por concurso y con participación de las comunidades en el jurado. En cada nodo se designó un promotor indígena, que estaría a cargo del relevamiento de contenidos y un promotor técnico que tomaría la responsabilidad de la capacitación. En Buenos Aires, se designó un líder indígena del proyecto y se contrató un webmaster para el diseño del sitio de Internet. También se previó el dictado de cursos de computación en Buenos Aires y La Plata, para la capacitación de indígenas de todo el país residentes en la ciudad de Buenos Aires y su conurbano.

\section{El sitio Puel Mapu We Dungun www.lofdigital.org.ar}

Puel Mapu We Dungun fue el nombre elegido para el sitio y significa "nuevas formas de comunicarnos en la Tierra del Este". Su dirección lofdigital alude al nombre de las comunidades en mapuzungun; significa por lo tanto: comunidad digital.

8 Coordinadora: Leonor Slavsky; Asesor Pedagógico: Osvaldo Cipolloni; Líder Indígena: Ignacio Prafil; Web master: Victoria Vidal. Nodo Esquel: Promotora Indígena (PI) Norma Nahuelquir; Promotora Técnica (PT) Verónica Botto; Nodo Viedma: PI Eduardo Cañulaf PT Yanina Tordi; Nodo Bahía Blanca: PI Pirén Curipán PT César Loncopan; Docentes informáticos Buenos Aires y La Plata: Pablo Martínez López y Victoria Vidal. 
Recogiendo el desafío de los Foros Internacionales se buscó lograr una página Web que resulte de un proceso de creación colectiva de conocimientos, que progresivamente las comunidades logren control técnico y social sobre las Tic's y que se transforme en un instrumento de investigación, creación y reinvención de la tradición, establecimiento de redes y reforzamiento de la identidad.

¿Q ué contenidos debía recoger el sitio Web? Los conocimientos tradicionales del pueblo mapuche en sus diferentes versiones; aquellos de los que el pueblo mapuche ha podido apropiarse, producir y reproducir y que forman parte de la cultura propia; los elaborados sobre el pueblo mapuche y las lecciones aprendidas por otros en la aplicación de un conocimiento mapuche; e información general de interés para el desarrollo social, cultural, económico y político local.

La página se diseñó en Buenos Aires luego de una discusión intragrupal sobre la mejor forma de organizar los contenidos. Las distintas entradas permiten navegar el sitio desde la Página de Inicio, con las novedades; Proyecto recoge información sobre distintos aspectos operativos de los nodos y los productos de la capacitación; Ad Mapu sobre conocimiento tradicional (con diversas entradas); Wall M apu (la tierra en constante movimiento) con noticias de actualidad y agenda; Winka, que provee información desde el punto de vista indígena sobre los acontecimientos posteriores a la conquista de la Patagonia, material académico producido sobre los mapuche que se encuentra en la W eb, entrevistas a investigadores y documentos públicos; Enlaces ofrece links a sitios de interés (otras pági nas mapuches, organismos nacionales e internacionales, fuentes de financiamiento para proyectos locales); Tecnología brinda información y capacitación informática y un Foro de discusión.

Se utilizaron tecnologías O pen Source (sin costo de licencias) con Servidor Linux y sistema de Blogs (Blogs W ordpress). Este sistema de administración de contenidos, de acuerdo al principio de descentralización, permite libertad para que los nodos puedan levantar sus propios contenidos y asimismo provee capacidad de interacción y diálogo con los lectores como veremos más adelante.

La página tiene una alta visitación. D esde 1/01/2006 al 31/10/2006 se recibieron 21.150 visitas y 64.660 fueron las páginas vistas. 


\section{La capacitación}

Los cursos de capacitación informática se llevaron adelante en todos los nodos a pesar de los problemas que encontramos en la conectividad. Contamos en Bariloche con el apoyo de la Universidad $\mathrm{N}$ acional del Comahue, en Bahía Blanca con la de la U niversidad $\mathrm{N}$ acional del Sur y en La Plata con la de la U niversidad de la Plata y el Laboratorio de Investigaciones en Antropología Social. Los cursos de Buenos Aires se desarrollaron en la sede del INET y en colegios secundarios, en Viedma en el Centro de Educación Media 19, en Esquel en la Escuela de Nivel M edio No 713 Juan Abdala Chayep y en la Escuela de Nivel Medio № 726 Padre U rbano Salort en El Maitén.

Partimos de la premisa de que los alumnos no cuentan con PC en sus casas ni en sus lugares de trabajo, por lo cual había que romper con el concepto de Personal Computer. Al no tener dispositivos de almacenamiento masivo de información personalizada, surgió la necesidad de utilizar a la propia Internet como el espacio para crear una PC virtual que permitiera acceder a la información personal (agenda, documentos, imágenes, páginas favoritas, mails, notas, RSS), desde cualquier computadora. Los cursos de usos de las TICS para jóvenes indígenas pretenden superar la mera introducción al uso técnico de la herramienta Internet. El objetivo es transformarlas en instrumento de apoyo y fortalecimiento cultural y comunitario. Los cursos abarcan desde el uso básico de una PC hasta el desarrollo de proyectos de investigación, utilizando Internet y sus herramientas de trabajo colaborativo. Se hizo hincapié en la búsqueda inteligente en la Web, búsqueda de información para la resolución de problemas concretos, correo electrónico y chateo y en la creación de blogs. Pueden verse los blogs creados por los nodos Buenos Aires y La Plata en http://w ww. lofdigital.org.ar/proyecto/cursos.php.

En los talleres realizados en Buenos Aires y con la colaboración de instituciones especializadas, los promotores técnicos e indígenas recibieron capacitación en las posibilidades de la educación y el trabajo a distancia9.

9 Las conferencias fueron brindadas de manera personal y en diferentes ámbitos por docentes profesionales del Programa Caminando Utopías de la Universidad de Buenos Aires (UBA), del Centro Blas Pascal I+DTE - Buenos Aires - Universidad Católica Argentina (UCA) y Educ.ar perteneciente al Portal Educativo del Gobierno Argentino. 
Leonor Slavsky

\section{Los logros y los obstáculos}

Logros

El "lofdigital" está lejos de ser el único sitio mapuche en la Web. De hecho este es el pueblo indígena de la Argentina y Chile que mayor producción posee en Internet. Algunos de ellos son medios periodísticos como los periódicos Azkintue www .azkintuwe.org y M apuexpress www .mapuexpress.net, otras son páginas personales de difusión cultural y emprendimiento comercial como la de la artesana platera Silvia Rinque www.silviarinque.com.ar, cursos de lengua a distancia www.cursomapunzugun.cl e incursiones en los blogs www.chedungun.blogspot.com.

Lo que caracteriza a la página W eb que nos ocupa es la gestión participativa en la producción de los contenidos culturales digitales. Los mismos se producen mayoritariamente a través del trabajo de los promotores en los nodos y la tecnología de los blogs permite un diálogo constante con los lectores y usuarios de la página. La visitación mapuche se ha incrementado notablemente en los últimos meses y le imprime la dirección a los contenidos. A pesar de tener en los Foros y en las $\mathrm{N}$ oticias de W all M apu información sobre temas políticos vinculados fundamentalmente a la recuperación y regularización territorial, a diferencia de otras páginas de fuerte contenido político, el lofdigital se ha orientado hacia la recuperación de contenidos culturales y de la identidad. También se ha ido construyendo como un referente para la reconstrucción del tejido social. Los temas más frecuentados en el Foro son: Lengua (y dentro de esta categoría, con más de 4.000 visitas el tema nombres mapuche, tanto por miembros del pueblo como por no mapuche), seguido de Cultura M ilenaria e Historia, mientras que otros como Recuperación de Territorio y Legislación carecen de visitas significativas. Debemos, no obstante, reconocer que los lectores no siempre respetan la categoría, insertando sus inquietudes personales que muchas veces la superan. Citaremos con cierta extensión algunos ejemplos que dan cuenta de la variedad de aproximaciones, algunas conciliatorias y otras muy críticas que se dan cita en la página (respetando la grafía original):

\section{Categoría Lengua:}

"hola hermanos soy ruben omar bilich hijo de jladys antieco, nieto de carmen antieco y vis nieto del casique andres antieco. ayer 
me senti orgulloso por encontrar esta web y un foro donde mi pueblo puede, opinar y bolcar sus inquietudes. por estos pagos hay muchos hermanos que mantienen viva la cultura, unos de los problemas mas comunes que me a tocado en carne propia es que el winca lamentablemente continua discrminando a quien diga que es decendiente de aborigen, y eso duele, pero como muchas veces le he dicho a mi abuela el winca no tiene la fuerza de voluntad que tenemos nosotros ni la fe. con esto no quiero despertar odio, contra el winca, pero aprendi a ver una realidad que por hay la sufren todos los pueblos aborigenes de argentina y latinoamerica. he aprendido poco de la lengua de mi pueblo, por hay muchos ancianos de trelew decendientes de mi pueblo, no hablan por la razon que muchas veces se han burlado de ellos, algo que le a pasado a mi abuela carmen. seria bueno que un dia la lengua ariginal de esta tierra se enceñara en cada colegio, como aci tambien la verdadera historia de mi pueblo no las mentiras que me enseñaron a mi. hermanos mios llegara el dia que el mapuche tehuelche reclamara con gritos de montañas, lo que le pertene por herencia, y volveremos a glorificar nuestra tierra. hoy debemos esperar con la pasiencia del tiempo, el viento traera a nuestras manos, lo que el winca nos quito. un amanecer de playas doradas y cordilleras nevadas, nos encontrara unidos como pueblo, como hermanos que somos. Ilevo un apelledo extranjero que no me pertenece, por que en mis venas la sangre mapuche reclama, mi herencia, la tierra, el viento, la nieve de los inviernos, el sol de los veranos pasajeros. hermanos no sientan verguenza y pidan a los ancianos que enceñen nuestra lengua, que es la lengua de la tierra. mapu tierra, che hombre. un habrazo ruben"

"Hola soy hijo de Azuncion Maria Railef bisnieta del cacique Jose M aria Railef nacida en O lacoaga. la barrancosa -bragado quisiera contactarme con familiares del cacique mi email es armandoromero@hotmail.com, desde ya muchas gracias"

"hola: en primer lugar quiero aclarar que no soy mapuche, soy de otro pueblo (mbya guaraní) soy nieta de un cacique de mi comunidad, hace 7 años que vivo acá en bs as . . con respecto a tú pregunta. cada pueblo son diferente entre sí por lo tanto también los nombres se dan de distintas manera. los guaraníes le ponen 
el nombre cuando los chicos cumple 1 o 2 años de vida, el que ponen los nombres es el jefe espiritual de la comunidad o sea el (opyguá ) cada nombre tiene su significado en el contexto sagrado y está muy relacionado con la naturaleza. las personas de la comunidades por lo general no pregunta al opyguá el significado de su nombre, ya que tienen el mayor de los respeto hacia esta persona lo cual tampoco esta prohibido hacerla. como es algo natural para nosotros. espero responder por lo meno a una parte de tú pregunta".

\section{Categoría Historia}

"Deseo tener información urgente sobre Federico Nahuelquir o de alguien muy cercano a él, o que me indiquen un teléfono para comunicarme. H ace 22 años adopté a una niña llamada M odesta Avelina Nahuelquir en General Roca y él figura como padre. Ella ahora tiene 26 años y quiere saber de su familia y la historia de sus ancestros, por supuesto que tiene otro nombre y apellido. Q uiero ayudarla porque el tema está afectando a su vida hasta que no tenga noticias. Gracias".

"Me gustaría saber el significado de mi apellido y de ser posible mi ascendencia. Mi abuelo materno creo que se llamaba Domingo Puelman y mi abuela materna era de apellido Curiche o Curicha, de la zona de Positos de Q uichaura. La hermana de mi abuelo falleció hace poco, era la menor de los hermanos Puelman, se Ilamaba Margarita Puelman y sabia muchísimo de la cultura mapuche. Vivía en Comodoro Rivadavia".

"Mari mari kom pu che,iñche Juan Marileo piñgen Allen waria mew tuw vn,mi nombre esjuan Marileo soy de Allen ,Provincia de Rio N egro, pertenesco a la comunidad Mapuche urbana NEWEN KOM queria responder al peñi millañanco que busca algún dato sobre posibles parientes, quiero informarle que aquí en Allen conosco a un chico de apellido millañanco y su papa tambien tiene parientes en Alumine, cualquier cosa dejo mi direccion electronica es; newmarilew@yahoo.com.ar".

\section{Categoría Cultura Milenaria}

"Lemoria tufachi we Chripan Antu, Kume Newentuaiñ kom MapuChe tufachi Chripantu, Trepengueiñ ka Wichran taiñ Newen 
Entre los dias 20 y 21 de junio (segun kalendario oxidental i kristiAno) es ke se han renovado las Fuerzas puNewen en nuestro Territorio Ancestral los MeliW ichranMapu, komo asi tambien en todo el hemisferio sur, ha sido la mitad del invierno, la noche mas larga, i el komienso o el re nacimiento de un nuevo despertar d la $\mathrm{N}$ aturaleza i todos sus elementos de todos los N ewen animales, plantas, cerros, Rios, Piedras, aves, Personas".

"Al iwal ke nuestros KuifiChe iem nuestros Antepasados, en diferentes partes del Territorio Ancestral MapuChe se ha konmemorado i festejado este akontecimiento, kon kisas algunas diferencias de dias en las juntas konsekuencia del awinkamiento i la invasion, pero kon un mismo objetivo, el de rekuperar las fuerzas i levantarnos en un nuevo amanecer universal, aunke el hecho especifiko ha sido el 20 i 21 en todos estos dias nos hemos juntado i kompartido mudai, chicha, ilo, kofke, o kisas solo un guiso, tambien historias, sueños, ideas, alegrias, penas, abrasos i N gueIlipun, a traves de Kuchralwe fogones prendidos en kada lugar, i del tradisional e importante baño en las vertientes, rios, lagos, arroyos, de kada Lof, para kurar nuestros males, para limpiarnos del WezaN ewen i renovar nuestro KumeN ew en i el kompromiso kon N uestros KuifiChe i kada Newen de Nuestro WalMapu WenuMapu Newen mulei NagMapu Newen mulei ka MincheMapu N ewen mulei, kada M apuChe konciente, ha rekordado i recibido esta Renovacion kon los suyos o kisas solo, en klandestinidad, preso, kon sus familias, en traun, en konflikto, pero en el fondo todos juntos, kon kada Kuchral i su humo, kon kada afafan, kon kada baño, kon kada N guellipun, nos hemos unido i akompañado, para muchos este sera un año dificil, de konfliktos, de tristesas, komo lo han generado los winka desde el gran despojo, pero a pesar de todo Mulei N ewen la fuersa esta, i nos akompaña, en kada uno, en kada rio, arbol, fuego,pajaro,,., Sabemos ke esto a sido asi desde la imposicion de su inM undo, pero tambien sabemos ke d Nuestro Lado estan nuestros grandes Toki iem, Kona iem, Lonko iem, Machi iem, ichrokom ReKumeChe iem, i kada Newen, por eso, no solo el deseo d un KumekeChripantu sino el kompromiso de Luchar junto a kada MapuChe, de Fortaleser nuestros lasos kortados, de Rekuperarnos, en este gran Mingako de Rekonstruxion, donde haya un MapuChe de Pie ahi estaremos, peleando por la Libertad i por sakar de nuestro Kamino el We- 
Leonor Slavsky

zakeN ewen ka Wekufe, por limpiar de nuestro Espiritu las enfermedades oxidentales komo el alkolismo i la drogadixion, komo la sumision i la traicion, komo la violencia interna i el machismo, i por Reenkontrarnos en FutaKumekekawin en FutaTraun de Libertad!! Kume WiñoiTripantu kom MapuChe, Chaeltumai Mañum puN ewen ka KuifiAlwe iem, Amulei WezaN ewen Kvpangue KumekeN ewen Fenchem Kume N ewentuaiñ, Kume MapuChe Kuzau N ieiñ, Kume MapuChe Kulliñ nieiñ, Kumeke Che muleiñ tain WalMapu ka Amulei tati wezache ianakonas ka winka, Weuaiñ Inchiñ!!!!!!!!!!!!!!!!!

Desde el NawelWapi Lafken PuelMapuChe, un Abrazo ke pretende ser Guerrero.... hai nos olemos, ,.,., Lemoria ka Peuaiñ".

Por otro lado, los comentarios a los blogs se muestran como una vía más directa e inmediata de comunicación. A modo de ejemplo, comentario del 2/12/2006 a la noticia del recital de Víctor Heredia:

"Hola, soy maestro de escuelas de la provincia de Buenos Aires y trato de guiar a mis alumnos en el conocimiento y la defensa de nuestros pueblos originarios, y me enorgullezco por ello. Quería comentar que anoche estuve en el teatro Ó pera y disfruté enormemente de Taki O ngoy, pero más allá de eso, me emocionó muchísimo la actuación de los hermanos de los diferentes pueblos. No tengo palabras para explicar los sentimientos que me abordaron en ese momento. Un abrazo enorme del corazón a todos los hermanos y seguiremos desde nuestro pequeño lugar acompañando la lucha por los derechos."

Comentarios entre el 25 y el 29 de junio con motivo de una nota acerca del We Tripantu:

"Feliz año, agredecidos toda la flia Liempe por poder comunicarnos En Epuyen este año Nuevo hubo festejos entre el pueblo mapuche. Es un buen comiezo.

"Kumei Trypantu i

Fentren Newen.

- Q ue sea un buen año para todos, especialmente para el Pueblo Nación Mapuche. Que se cumplan muchos de los deseos, que motivan esta diaria lucha. Q ue nos encuentre unidos.

Walter Kona Niyeu-Somuncura". 
Keme tripantü pu peñi, pu lamnien, pu huinca!! Buen año para todos/as y cuando digo para todos incluyo pueblo mapuche y no mapuches. Tratemos de terminar con la discriminación. No hagamos el "ojo por ojo diente por diente" Conservar nuestra cultura a través del respeto.- Buen año!! Atte Alejandro N ahuel.- Sta. Rosa (La Pampa)".

La página se construye en la multivocalidad. Reúne material producido por los propios actores sociales mapuche (promotores de los nodos) tales como artículos, entrevistas, noticias, enlaces a otros sitios de interés, y en el sector W inka reseñas de libros y artículos académicos sobre los mapuche que se encuentran on line, todos ellos con la posibilidad de recibir comentarios de los lectores/visitantes/colaboradores, indígenas y no indígenas. De este modo se ha dislocado el rol sobre el control de "autenticidad" y la validación de los contenidos, que no está en manos del profesional antropólogo, sino que lo posee la propia audiencia del sitio a través de sus comentarios. U n salto cualitativo importante se produjo en los últimos días, cuando un visitante de la página se convirtió en cronista proponiendo un artículo sobre cosmovisión "Trem Trem Kay Kay Filú" que fue levantado a la página http://www.lofdigital.org.ar/admapu/?p=151.

O tros logros en los dos años de trabajo son los contactos comerciales a comunidades y de apoyo a emprendimientos y proyectos, más de cien jóvenes capacitados en los distintos nodos y el haber sido ternados para el premio Mate. $\operatorname{ar}^{10}$ dentro de la categoría patrimonio cultural.

La meta final del proyecto es lograr la apropiación social de las tecnologías, esto es, comprender que la conectividad no es un fin en sí mismo, sino una herramienta que puede ayudar a construir soluciones concretas para los problemas y necesidades de la gente. Deberíamos llegar al punto en que la gente

"pueda preguntarse primero ¿qué deseo resolver? Y una vez respondido esto, tenga los recursos y conocimientos suficientes para responder ¿cómo puede ayudarme Internet a lograrlo?, hacer un uso efectivo de esta tecnología y lograr la solución del problema planteado (en este sentido, se va más allá del uso mismo de la tecnología porque se establece una relación explícita y permanente

10 Concurso que consagra a los mejores sitios web argentinos según la categoría o rubro al que pertenecen www.mate.ar.org. 
Leonor Slavsky

entre la realidad en la que se vive y la utilización de la tecnología)" (Del Alamo 2003).

Los obstáculos

Podemos clasificar a los obstáculos para el desarrollo del proyecto en externos e internos. Entre los primeros, corroborando el informe de Hernández y Calcagno (2003), la desigual situación con relación a la conectividad que se verifica en el país y que sigue la lógica del mercado y no la de las necesidades sociales. Según expresiones de dirigentes de la empresa Telefónica, la instalación de banda ancha en poblaciones pequeñas no redunda en rentabilidad para la empresa, de modo que localidades como El Maitén están sujetas a emprendimientos privados que sólo proveen al pueblo a alto costo. De allí a las comunidades circundantes como Cushamen o Ñ orquinco se torna prácticamente imposible por la desigual topografía del terreno, que necesita de la instalación de antenas para retransmitir la señal. La provincia de Chubut posee un satélite que está en condiciones de conectar a toda la provincia por esa vía, pero a pesar de los proyectos, todavía no se ha realizado.

Ciudades de tamaño medio como Viedma tienen una capacidad limitada, que obliga a más de un año de espera el cupo para conectar la escuela secundaria donde se realiza el curso de capacitación. Recordemos que el servicio de Internet fue declarado de interés nacional por decreto $\mathrm{N}$ o 554/97. Hace pocos días atrás, el diario Clarín (5/12/2006) publicó un fallo que afirma que las computadoras no se pueden embargar por ser "indispensables" para acceder a Internet. Los jueces entendieron que el acceso a la Web es "primordial para desenvolverse en la vida actual".

Ante la evidencia de que la mayoría de las comunidades y las escuelas con población mapuche carecen de acceso a Internet (pero muchas poseen computadoras), estamos elaborando una versión navegable de la página en CD para ser distribuida en las escuelas de la Patagonia.

O tro factor es el tiempo de desarrollo del proyecto. Los financiamientos internacionales, que en nuestro país no suelen contar con contraparte nacional y que tienen plazos limitados para su ejecución, no autorizan un acompañamiento prolongado que permita la consolidación de los logros. En esta última etapa del proyecto (lapso del fi- 
nanciamiento: febrero de 2005 a febrero de 2007), estamos abocados a la elaboración conjunta de una estrategia de asociación con instituciones locales y regionales que aseguren la sustentabilidad a futuro.

Entre los obstáculos internos encontramos, como en todo pueblo complejo y numeroso, ideologías étnicas en conflicto. Estas se verifican en su relación con el Estado-Nación y en su concepción del patrimonio. Así, mientras al gunos grupos y organizaciones ven con beneplácito la posibilidad de conexión con el exogrupo y lo visualizan como una posibilidad para el reforzamiento de la identidad, particularmente entre los jóvenes, para otros se trata de una nueva estrategia colonial de expoliación. Ejemplo de ello fue la reacción del director mapuche de la escuela de Cushamen quien negó toda colaboración a la página, aduciendo la propiedad intelectual del conocimiento mapuche y el riesgo de ponerlo en dominio público. Asimismo, nos comentó que instruyó a su hija (alumna del curso de capacitación en El Maitén) que "si le preguntaban, contestara que no sabía".

Otros argumentos tienen que ver con la desconfianza dentro de las comunidades por los efectos disruptivos de las Tics en las pautas sociales y culturales y también con la fuente del financiamiento, dados los conflictos con las empresas mineras canadienses particularmente en la zona de Esquel. Las luchas por el poder local entre las distintas organizaciones indígenas también actúa como factor paralizante y obligó a discontinuar el nodo Bariloche.

\section{Conclusiones}

Recorrimos en las páginas de este artículo una serie de temas que necesariamente deben encontrar su punto de contacto en estas conclusiones. Partimos del reconocimiento del cambio, producido en las últimas décadas, en las sociedades indígenas y sus reclamos de autonomía y autoatribución de identidad. Establecimos los niveles de conflicto que estos reclamos generan, con relación a la antropología como disciplina especializada en el estudio de la alteridad y con el rol del antropólogo como perito, en el establecimiento de la autenticidad de culturas y tradiciones. Vimos también cómo en la sociedad globaliza$\mathrm{da}$, que propicia la reemergencia de identidades étnicas a lo ancho del planeta, los pueblos indígenas encontraron un nicho apropiado para la expresión de sus demandas, y cómo la Internet y el correo electrónico resultaron aliados de estos procesos. 
Los pueblos aborígenes tienen algo que decir con relación a la Sociedad de la Información y lo han hecho en múltiples Foros Internacionales. El proyecto que se ha descrito, se inscribe en este marco referencial buscando devolver la palabra a nuestros tradicionales "objetos de estudio". Es pertinente recordar lo expresado por Bauman citado en el trabajo de Paine:

"Zygmunt Bauman (...) considera que la autenticidad tiene tanto que ver con lo que somos como con lo que seremos o deberíamos ser. (...) Esto se aplica bien a las visiones de las sociedades coloniales sobre sí mismas como siendo o deviniendo en "un pueblo". El costado petulante de esta versión es el que carga a la aboriginalidad con una autenticidad que tiene que ver con el pasado. Sin embargo, la visión que el colono tiene sobre el pasado aborigen varía. Así veremos contradicciones y aun ambivalencia entre un pasado que está desapareciendo y uno que debería ser rescatado. Estas contradicciones existen también en los indígenas, en la forma en que ven su futuro y quizás igualmente en los sentidos atribuidos al pasado, escindido entre un yo impuesto (por los otros) y de un yo "real". De estos sentidos, el primero debe ser exorcizado y el otro realizado nuevamente mientras se lo actualiza, pero ¿de que manera?" (Paine 2000:79-80).

No podemos entonces pensar la autenticidad ni como dada ni como inmodificable, sino como un proceso con cualidades relacionales y contingentes. En otras palabras, "lo auténtico" tiene fecha y no es inmune al cambio cultural aún cuando el sentido común diga que si es auténtico es "genuino", y por lo tanto no "ficcional" en el sentido atribuido por Hobsbawm y Ranger (1987). El argumento dominante para Friedman, es que las realidades culturales son siempre producidas en contextos sociohistóricos precisos y que es necesario tener en cuenta el proceso que genera esos contextos, para dar razón de la naturaleza de la práctica de la identidad y la producción de esquemas históricos. Esto incluye tanto las identificaciones "inventadas por los antropólogos" como también las de los pueblos que nos ocupan (Friedman 1992).

La apropiación social de las tecnologías de la información y la comunicación, brindan posibilidades que superan la simple producción de contenidos culturales en las áreas de educación (e-learning), salud (telesalud), actividad económica (e-commerce), difusión y promoción de sus demandas y derechos. 
¿Cuál es el rol que compete a la academia y al profesional en ciencias sociales? D esde la perspectiva de una antropología vinculada a la gestión, un abordaje metodológico que permite la incorporación de las voces, perspectivas y recursos de los pueblos indígenas, es el abanico de formas que con diferentes nombres (investigación participativa, investigación-acción) retoman los trabajos pioneros de Paulo Freire y O rlando Fals Borda en América Latina y Kurt Lewin en Estados U nidos. En nuestro medio todavía resulta novedosa la aplicación de estas metodologías en el campo antropológico. Retomando la pregunta inicial, diremos que son una herramienta eficiente para trabajar sobre temas de interés antropológico, con los indígenas que forman parte del equipo de investigación y gestión, para la obtención de materiales útiles que permitan avanzar en el camino de la autogestión cultural, la autodefinición de la identidad étnica, la posibilidad de pensar nuevos roles para el antropólogo como profesional y reflexionar críticamente sobre la relación teoría/práctica sin la cual "la teoría se convierte en palabrerío y la práctica en activismo" (Freire 2003: 24).

Los pueblos indígenas no desean permanecer anclados en el pasado. La identidad no se relaciona con el cambio tecnológico sino con la conciencia histórica, el sentido de pertenencia y el deseo de continuidad del pueblo. Según las palabras del dirigente mapuche Jorge Nahuel: "Q ueremos ser hombres modernos del siglo XXI, conservando nuestra identidad" (D ocumento final 2002).

\section{Bibliografía}

DEL ÁlAMO O O . (2003) “Esperanza tecnológica: Internet para los pueblos indígenas de América Latina", en: Revista Instituciones y Desarrollo, № 14-15, Barcelona, Instituto Internacional de Governabilitat de Catalunya, pp. 43-79.

BELL, D. (1977) Las contradicciones culturales del capitalismo. México, Alianza.

BENASSINI FELIX, C. (2004) "De sociedad de la información a comunidades de la información: tres momentos para su llegada", en: Revista Mexicana de Comunicación, № 84, México, Fundación Buendía, disponible en Internet en: http:// www.mexicanadecomunicacion.com.mx/Tables/RM C/rmc84/ momentos.html. 
Leonor Slavsky

BONFIL BATALLA, G. (1989) "La teoría del control cultural en el estudio de los procesos étnicos", en: Arinsana, Caracas N o 10, pp. $5-36$.

COLOMBO VILARRASA, C. (2005) "Participación ciudadana en la red", en: Boletín Gestión Cultural, Participación Ciudadana, № 11, Instituto de Gobernabilidad de Catalunya disponible en Internet en: http://www.gestioncultural.org/gc/boletin/2005/bgc11participacion.htm\#forocentral.

DOCUMENTO FINAL (2002) El Universo Cultural Mapuche: El $\mathrm{N}$ guillatun, ceremonia religiosa en Argentina y Chile. BuenosAires, Instituto Nacional de Antropología y Pensamiento Latinoamericano (inédito).

FREIRE, P. (2003) Pedagogía de la Autonomía; saberes necesarios para la práctica educativa. Buenos Aires, Siglo XXI.

FRIEDMAN, J. (1992) "The Past in the Future: History and the Politics of Identity", en: American Anthropologist, № 94 (4), pp. 837-859.

GREENWOOD, D. J. y M. LEVIN (1998) Introduction to Action Research; Social Research for Social Change. London, Sage.

HERNÁNDEZ, I. y S. CALCAGNO (2003) Los Pueblos Indígenas y la Sociedad de la Información en América Latina y el Caribe Un Marco para la Acción. Santiago de Chile, CEPAL.

HO BSBAW M, E. y T. RAN GER (eds.) (1987) The Invention of Tradition. Cambridge, Cambrige U niversity Press.

KLESERT, A. L. y L. BENALLIE Jr. (1994) "Navajo Nation Archeology Department. Commentary: Indians and Anthropologists", en: Practicing Anthropology, Vol. 16, № 2 .

MASU DA, Y. (1984) La Sociedad Informatizada como Sociedad PostIndustrial. M adrid, Fundesco-Tecnos.

M ATTELART, A. (1977) Multinacionales y sistemas de comunicación: los aparatos ideológicos del imperialismo. M éxico, Siglo XXI.

- - (2000) Historia de la utopía planetaria. De la ciudad profética a la sociedad global. Barcelona, Paidós.

PAINE, R. (2000) "Aboriginality, authenticity and the Settler world", en: A. P. COHEN (ed.) Signifying Identities, Anthropological 
perspectives on boundaries and contested values. London, Routledge.

SLAVSKY, L. (1998) "El patrimonio de los pueblos indígenas", en: Actas del I Congreso Internacional sobre Patrimonio Histórico e identidad cultural. Tucumán, CRISCO S-U NSTA, pp. 185-189.

- - (2004) "Memoria y patrimonio indígena. Hacia una política de autogestión cultural mapuche en Río Negro", en: VII Congreso Argentino de Antropología Social, Villa Giardino, Simposio: Políticas Culturales y la gestión de la identidad y la memoria.

- (2005) "Autogestión de la identidad y la memoria en la Patagonia Argentina", en: Boletín Gestión Cultural, Participación Ciudadana, Instituto de Gobernabilidad de Catalunya, № 11, disponible en Internet en: http://www.gestioncultural.org/gc/boletin/pdf/bgc11LSlavsky.pdf.

_- (2006) "Tecnologías de la información y la comunicación para jóvenes mapuche", en: Novedades de Antropología, Año 15, № 54, Buenos Aires, IN APL, pp. 12-13.

\section{Resumen}

Analizamos en este trabajo el desarrollo del proyecto "Tecnologías de la Información y la Comunicación para Jóvenes Mapuche" y su contexto de emergencia, en el marco de las Cumbres Internacionales sobre la Sociedad de la Información y su relación con los procesos de construcción de la identidad étnica. Se discuten los conceptos de autenticidad cultural, la nueva relación entre los pueblos indígenas y la academia, y el rol del antropólogo en los proyectos de gestión.

Palabras claves: Pueblos indígenas, TIC, identidad étnica, autenticidad, rol antropólogo.

\section{ICT for young indigenous people: Creating collective digital cul- tural contents a Mapuche experience \\ Summary}

In this paper we analyze "Information and Communication Technologies for Young Mapuche People" and the context in which they emerged, related with the International Summits on the Society of Information and how it relates with ethnic identity construction proc- 
Leonor Slavsky

esses. We discuss the concept of cultural authenticity, the new relationship between indigenous peoples and the academic world, and the anthropologist's role in applied social projects.

Key words: Indigenous Peoples, ICT, ethnic identity, authenticity, anthropologist's role.

Recibido: 09/02/07, aceptado: 02/03/07. 